\title{
Optimality conditions for a bilevel optimization problem in terms of KKT multipliers and convexificators
}

\author{
Nazih Abderrazzak Gadhi ${ }^{1, *}$, Lahoussine Lafhim ${ }^{1}$ \\ 1 Department of Mathematics, Sidi Mohamed Ben Abdellah University \\ Dhar El Mahraz, Fes, Morocco \\ E-mail: 〈ngadhi@hotmail.com,lafhim68@gmail.com〉
}

\begin{abstract}
In this paper we investigate a bilevel optimization problem by using the optimistic approach. Under a non smooth generalized Guignard constraint qualification, due the optimal value reformulation, the necessary optimality conditions in terms of convexificators and Karush-Kuhn-Tucker (KKT) multipliers are given.
\end{abstract}

Keywords: bilevel optimization, constraint qualification, optimality conditions

Received: August 25, 2019; accepted: September 24, 2019; available online: December 13, 2019

DOI: $10.17535 /$ crorr.2019.0026

\section{Introduction}

Using the optimistic approach in bilevel optimization, where we assume that the leader presupposes cooperation of the follower in the sense that the latter will choose in each time that solution in the solution set of the follower's parametric optimization problem which is best suited with respect to the leader's objective function, we investigate the following bilevel optimization problem

$$
(P) \quad \min _{x, y} F(x, y) \text { s.t. } \quad G_{j}(x, y) \leq 0, j \in J, \quad y \in \psi(x),
$$

where, for each $x \in \mathbb{R}^{n_{1}}, \psi(x)$ is the set of optimal solutions of the following parametric optimization problem

$$
\min _{y} f(x, y) \quad \text { s.t. } g_{i}(x, y) \leq 0, \quad i \in I
$$

where $F: \mathbb{R}^{n_{1}} \times \mathbb{R}^{n_{2}} \rightarrow \mathbb{R}$ and $g_{i}: \mathbb{R}^{n_{1}} \times \mathbb{R}^{n_{2}} \rightarrow \mathbb{R}, i \in I=\{1, \cdots, q\}$ are locally Lipchitz functions, $f, G_{j}: \mathbb{R}^{n_{1}} \times \mathbb{R}^{n_{2}} \rightarrow \mathbb{R}, j \in J=\{1, \cdots, p\}$ are convex continuous functions and $n_{1} \geq 1, n_{2} \geq 1, p \geq 1, q \geq 1$ are integers. The point $\bar{u}=(\bar{x}, \bar{y})$ is said to be a local optimal solution of $(P)$ if it is an optimal solution of the problem

$$
\left\{\begin{array}{r}
\text { Minimize } F(x, y) \\
\text { subject to }:(x, y) \in E,
\end{array}\right.
$$

where

$$
E=\left\{\begin{array}{c}
(x, y) \in \mathbb{R}^{n_{1}} \times \mathbb{R}^{n_{2}}: \\
G_{j}(x, y) \leq 0, g_{i}(x, y) \leq 0, i \in I, j \in J, y \in \psi(x)
\end{array}\right\}
$$

${ }^{*}$ Corresponding author. 
A lot of research has been carried out in bilevel optimization problems $[1,3,4,5,6,8,17,18$, 19, 20]. Ye and Zhu [19] give optimality conditions without convexity assumption on the lower level problem and without the assumption that the solution set $\psi(x)$ is a singleton. Under semiLipschitz property, Zhang [20] extends the classical approach to allow the nonsmooth problem data; he derives existence and optimality conditions for problems in terms of a graph set of the solution multifunction to the lower-level problem.

In this paper, our approach consists of reformulating our problem using the optimal value function of the lower level problem and after investigating necessary optimality conditions of $(P)$. Our results are obtained, under a non smooth generalized Guignard constraint qualification, in terms of convexificators and Karush-Kuhn-Tucker multipliers.

The rest of the paper is organized in this way: Section 2 contains basic definitions and preliminary material from nonsmooth variational analysis, Section 3 addresses main results (optimality conditions), while main conclusion is given in Section 4.

\section{Preliminaries}

In this section, we recall some basic constructions and results from nonsmooth analysis. For a subset $D$ of $\mathbb{R}^{n}$, the sets $c l D$, conv $D, \overline{\text { conv }} D(=$ cl conv $D)$, cone $D, \overline{\text { cone }} D(=$ cl cone $D)$ and $D^{-}$stand for the closure of $D$, the convex hull of $D$, the closed convex hull of $D$, the convex cone generated by $D$, the closed convex cone generated by $D$ and the negative polar cone of $D$, repectively.

Let $D$ be a subset of $\mathbb{R}^{n}$ and $x \in \operatorname{cl} D$. The contingent cone $T(D, x)$ to $D$ at $x$ is defined by

$$
T(D, x)=\left\{v \in \mathbb{R}^{n}: \exists t_{n} \downarrow 0 \text { and } \exists v_{n} \rightarrow v \text { such that } x+t_{n} v_{n} \in D\right\} .
$$

A set-valued mapping $H: \mathbb{R}^{n} \rightrightarrows \mathbb{R}^{m}$ will be said to be inner semicompact at a point $\bar{x}$ with $H(\bar{x}) \neq \emptyset$, if for every sequence $x_{k} \rightarrow \bar{x}$ with $H\left(x_{k}\right) \neq \emptyset$, there is a sequence of $y_{k} \in H\left(x_{k}\right)$ that contains a convergent subsequence. The mapping $H$ is inner semicontinuous at $(\bar{x}, \bar{y}) \in \operatorname{gr}(H)$ if for every sequence $x_{k} \rightarrow \bar{x}$ there is a sequence of $y_{k} \in H\left(x_{k}\right)$ that converges to $\bar{y}$.

Proposition 1. Let $f$ be Lipschitz around $(\bar{x}, \bar{y})$ for every $\bar{y} \in \psi(\bar{x})[14]$

- If $\psi$ is inner semicompact at $\bar{x}$, one gets the Lipschitz continuity of the value function $V$ defined by

$$
V(x)=\min _{y}\left\{f(x, y): g_{i}(x, y) \leq 0, i \in I, y \in \mathbb{R}^{n_{2}}\right\} .
$$

- If $\psi$ is inner semicontinuous at $(\bar{x}, \bar{y})$, one gets the Lipschitz continuity of the value function $V$.

Now, we recall the definitions related to convexificators given by Jeyakumar and Luc [10] and Dutta and Chandra [8]. Let $f: \mathbb{R}^{n} \rightarrow \mathbb{R} \cup\{+\infty\}$ be a given function and let $x \in \mathbb{R}^{n}$ where $f(x)$ is finite. The expressions

$$
\begin{aligned}
& f_{d}^{-}(x, v)=\liminf _{t \searrow 0}[f(x+t v)-f(x)] / t \\
& f_{d}^{+}(x, v)=\underset{t \searrow 0}{\limsup }[f(x+t v)-f(x)] / t
\end{aligned}
$$

signify the lower and upper Dini directional derivatives of $f$ at $x$ in the direction $v$, respectively.

Definition 1. The function $f: \mathbb{R}^{n} \rightarrow \mathbb{R} \cup\{+\infty\}$ is said to have an upper convexificator (UCF) $\partial^{*} f(x)$ at $x$ if $\partial^{*} f(x) \subseteq \mathbb{R}^{n}$ is closed and, for each $v \in \mathbb{R}^{n}$,

$$
f_{d}^{-}(x, v) \leq \sup _{x^{*} \in \partial^{*} f(x)}\left\langle x^{*}, v\right\rangle
$$


Optimality conditions for a bilevel optimization problem in terms of KKT multipliers and convexificators 331

Definition 2. The function $f: \mathbb{R}^{n} \rightarrow \mathbb{R} \cup\{+\infty\}$ is said to have a lower convexificator (LCF) $\partial_{*} f(x)$ at $x$ if $\partial_{*} f(x) \subseteq \mathbb{R}^{n}$ is closed and, for each $v \in \mathbb{R}^{n}$,

$$
f_{d}^{+}(x, v) \geq \inf _{x^{*} \in \partial_{*} f(x)}\left\langle x^{*}, v\right\rangle .
$$

A closed set $\partial^{*} f(x) \subseteq \mathbb{R}^{n}$ is said to be a convexificator of $f$ at $x$ if it is both an upper and lower convexificator of $f$ at $x$.

Remark 1. The convexificators are neither necessarily compact nor convex [7]. These relaxations allow applications to a large class of nonsmooth continuous functions. For instance, the function $f: \mathbb{R} \rightarrow \mathbb{R}$ defined by $f(x)=-|x|$, admits a non convex convexificator $\partial^{*} f(0)=\{-1,1\}$ at 0 .

The following definition has been proposed by Dutta and Chandra. For more details [8].

Definition 3. The function $f: \mathbb{R}^{n} \rightarrow \mathbb{R} \cup\{+\infty\}$ is said to have an upper semi-regular convexificator (USRCF) $\partial^{*} f(x)$ at $x$ if $\partial^{*} f(x)$ is an upper convexificator at $x$ and, for each $v \in \mathbb{R}^{n}$,

$$
f_{d}^{+}(x, v) \leq \sup _{x^{*} \in \partial^{*} f(x)}\left\langle x^{*}, v\right\rangle .
$$

Remark 2. The Clarke [2], Michel-Penot [13] and Murdokhovich [16] subdifferentials are upper semi-regular convexificators of $f$ when $f$ is a locally Lipschitz function. However, the convex hull of an upper semi-regular convexificator of a locally Lipschitz function may be strictly contained in both the Clarke and the Michel-Penot subdifferentials.

Definition 4. Let $f: \mathbb{R}^{n} \rightarrow \mathbb{R}$ be a locally Lipschitz function on $\mathbb{R}^{n}$. The Clarke subdifferential of $f$ at $\bar{x}$ is defined by

$$
\partial_{c} f(\bar{x}):=\left\{\eta \in \mathbb{R}^{n}: f^{\circ}(\bar{x}, v) \geq\langle\eta, v\rangle \forall v \in \mathbb{R}^{n}\right\} .
$$

where, for each $v \in \mathbb{R}^{n}$,

$$
f^{\circ}(\bar{x}, v)=\limsup _{x \rightarrow \bar{x}, t \searrow 0} \frac{f(x+t v)-f(\bar{x})}{t}
$$

is known as the Clarke generalized derivative of $f$ at $\bar{x}$ with respect to $v$.

Remark 3. For a locally Lipschitz function $F, \partial_{c} f(\bar{x})$ is a convexificator of $f$ at $x[10]$.

Let

$$
\Omega_{L}=\left\{(x, y) \in \mathbb{R}^{n_{1}} \times \mathbb{R}^{n_{2}}: g_{i}(x, y) \leq 0, i \in I\right\}, \quad I(x, y)=\left\{i \in I: g_{i}(x, y)=0\right\},
$$

and

$$
\Omega=\left\{(x, y) \in \mathbb{R}^{n_{1}} \times \mathbb{R}^{n_{2}}: f(x, y)-V(x) \leq 0, g_{i}(x, y) \leq 0, i \in I, G_{j}(x, y) \leq 0, j \in J\right\} .
$$

To proceed further, we shall need the following regularity conditions.

- We say that $(\bar{x}, \bar{y}) \in \Omega_{L}$ is lower-level regular if

$$
\left[\sum_{i \in I(\bar{x}, \bar{y})} \lambda_{i} v_{i}=0, \lambda_{i} \geq 0\right] \Rightarrow\left[\lambda_{i}=0 \text { for all } i \in I(\bar{x}, \bar{y})\right]
$$

whenever $\left(u_{i}, v_{i}\right) \in \partial_{c} g_{i}(\bar{x}, \bar{y})$ with some $u_{i} \in \mathbb{R}^{n_{1}}$ as $i \in I(\bar{x}, \bar{y})$. 
- We say that the nonsmooth Abadie constraint qualification holds at $(\bar{x}, \bar{y})$ if

$$
\left(\bigcup_{i \in I(\bar{x}, \bar{y})} \operatorname{conv}^{*} g_{i}(\bar{x}, \bar{y}) \cup \bigcup_{j \in J(\bar{x}, \bar{y})} \operatorname{conv}^{*} G_{j}(\bar{x}, \bar{y}) \cup \partial f(\bar{x}, \bar{y})-\partial V(\bar{x}) \times\{0\}\right)^{-} \subseteq T(\Omega,(\bar{x}, \bar{y})) .
$$

- We say that nonsmooth generalized Guignard constraint qualification holds at $(\bar{x}, \bar{y})$ if

$$
[T(\Omega,(\bar{x}, \bar{y}))]^{-} \subseteq \overline{c o n e}\left(\left(\bigcup_{i \in I(\bar{x}, \bar{y})} \partial^{*} g_{i}(\bar{x}, \bar{y})\right) \cup\left(\bigcup_{j \in J(\bar{x}, \bar{y})} \partial^{*} G_{j}(\bar{x}, \bar{y})\right) \cup(\partial f(\bar{x}, \bar{y})-\partial V(\bar{x}) \times\{0\})\right)
$$

Here, $\partial$ stand for the subdifferential of convex analysis.

Remark 4. The nonsmooth Abadie constraint qualification implies the nonsmooth generalized Guignard constraint qualification. The converse is not always true.

\section{Necessary optimality conditions}

For all the sequel, it is assumed that the leader presuppose cooperation of the follower in the sense that the latter will choose in each time that solution in $\psi(x)$ which is best suited with respect to the leader's objective function.

In this case, $(P)$ can be replaced by

$$
\left(P^{*}\right): \quad\left\{\begin{array}{c}
\text { Minimize } F(x, y) \\
\text { subject to }:(x, y) \in \Omega
\end{array}\right.
$$

provided that $\left(P^{*}\right)$ has an optimal solution [12], where for all $(x, y) \in \mathbb{R}^{n_{1}} \times \mathbb{R}^{n_{2}}$,

$$
V(x):=\min _{y}\left\{f(x, y): g_{i}(x, y) \leq 0, i \in I, y \in \mathbb{R}^{n_{2}}\right\} .
$$

Note that, since data are all convex, the optimal value function $V$ is also convex.

Remark 5. Under the following hypotheses $\left(H_{1}\right),\left(H_{2}\right),\left(H_{3}\right)$ and $\left(H_{4}\right)$, the optimization problem $(P)$ has at least one optimal solution $[9]$.

$\left(H_{1}\right): F(.,$.$) is lower semicontinuous (l.s.c.) on \mathbb{R}^{n_{1}} \times \mathbb{R}^{n_{2}}$;

$\left(H_{2}\right): f(.,$.$) is lower continuous, V($.$) is upper semicontinuous (u.s.c.) on \mathbb{R}^{n_{1}}$;

$\left(H_{3}\right): g_{j}(.,$.$) and G_{i}(.,$.$) are lower continuous on \mathbb{R}^{n_{1}} \times \mathbb{R}^{n_{2}}$;

$\left(H_{4}\right)$ : The problem $\left(P^{*}\right)$ has at least one feasible solution and its feasible set is bounded.

Especially, under these conditions, $\Omega$ is a nonempty compact set and $F$ is a lower semicontinuous function.

Theorem 1. Let $\bar{u}=(\bar{x}, \bar{y}) \in C$ be a local optimal solution of $(P)$. Assume that $F$ admits a bounded (USRCF) $\partial^{*} F(\bar{u})$ at $\bar{u}$, that $G_{j}, j \in J, g_{i}, i \in I$, admit (UCFs) $\partial^{*} G_{j}(\bar{u}), \partial^{*} g_{i}(\bar{u})$, respectively at $\bar{u}$. Suppose that the nonsmooth generalized Guignard constraint qualification holds at $(\bar{x}, \bar{y})$, that the solution-set-mapping of $S$ is inner semicompact at $\bar{x}$, and that for each vector $y \in \psi(\bar{x}),(\bar{x}, y)$ is lower-level regular. Then, there exist $y^{*} \in \psi(\bar{x}), \pi \geq 0, \xi \geq 0, \mu \geq 0$ and $\left(\lambda_{1}^{*}, \cdots, \lambda_{q}^{*}\right) \in \mathbb{R}_{+}^{q}$ such that

$$
\begin{aligned}
0 \in & \operatorname{conv} \partial_{x}^{*} F(\bar{x}, \bar{y})+\sum_{j \in J(\bar{x}, \bar{y})} \pi_{j} \partial_{x}^{*} G_{j}(\bar{x}, \bar{y})+\sum_{i \in I(\bar{x}, \bar{y})} \xi_{i} \partial_{x}^{*} g_{i}(\bar{x}, \bar{y}) \\
& +\mu\left(\partial_{x} f(\bar{x}, \bar{y})-\partial_{x} f\left(\bar{x}, y^{*}\right)-\sum_{i \in I} \lambda_{i}^{*} \partial_{x} g_{i}\left(\bar{x}, y^{*}\right)\right)
\end{aligned}
$$


Optimality conditions for a bilevel optimization problem in terms of KKT multipliers and convexificators 333

$$
0 \in \operatorname{conv} \partial_{y}^{*} F(\bar{x}, \bar{y})+\sum_{j \in J(\bar{x}, \bar{y})} \pi_{j} \partial_{y}^{*} G_{j}(\bar{x}, \bar{y})+\sum_{i \in I(\bar{x}, \bar{y})} \xi_{i} \partial_{y}^{*} g_{i}(\bar{x}, \bar{y})+\mu \partial_{y} f(\bar{x}, \bar{y})
$$

and

$$
0 \in \partial_{y} f\left(\bar{x}, y^{*}\right)+\sum_{i \in I} \lambda_{i}^{*} \partial_{y} g_{i}\left(\bar{x}, y^{*}\right) \quad, \quad \lambda_{i}^{*} g_{i}\left(\bar{x}, y^{*}\right)=0 .
$$

Proof. Since $(\bar{x}, \bar{y})$ is an optimal solution of $(P)$, it is an optimal solution of $\left(P^{*}\right)$. Let $\left(v_{1}, v_{2}\right) \in$ $T(\Omega,(\bar{x}, \bar{y}))$. Then, there exist $t_{n} \downarrow 0$ and $\left(v_{n_{1}}, v_{n_{2}}\right) \rightarrow\left(v_{1}, v_{2}\right)$ such that $(\bar{x}, \bar{y})+t_{n}\left(v_{n_{1}}, v_{n_{2}}\right) \in \Omega$ for all $n$. Since $(\bar{x}, \bar{y})$ is a minimum of $F$ over $\Omega$, one has

$$
\frac{F\left((\bar{x}, \bar{y})+t_{n}\left(v_{n_{1}}, v_{n_{2}}\right)\right)-F(\bar{x}, \bar{y})}{t_{n}} \geq 0, \quad \text { for sufficiently large } n .
$$

Remarking that

$$
\begin{aligned}
& \frac{F\left((\bar{x}, \bar{y})+t_{n}\left(v_{n_{1}}, v_{n_{2}}\right)\right)-F(\bar{x}, \bar{y})}{t_{n}} \\
= & \frac{F\left((\bar{x}, \bar{y})+t_{n}\left(v_{n_{1}}, v_{n_{2}}\right)\right)-F\left((\bar{x}, \bar{y})+t_{n}\left(v_{1}, v_{2}\right)\right)}{t_{n}}+\frac{F\left((\bar{x}, \bar{y})+t_{n}\left(v_{1}, v_{2}\right)\right)-F(\bar{x}, \bar{y})}{t_{n}}
\end{aligned}
$$

and that $F$ is locally Lipschitz, one deduces that

$$
\begin{aligned}
& F_{d}^{+}\left((\bar{x}, \bar{y}),\left(v_{1}, v_{2}\right)\right) \\
= & \limsup \frac{F\left((\bar{x}, \bar{y})+t_{n}\left(v_{1}, v_{2}\right)\right)-F(\bar{x}, \bar{y})}{t_{n}} \\
= & \limsup _{n} \frac{F\left((\bar{x}, \bar{y})+t_{n}\left(v_{n_{1}}, v_{n_{2}}\right)\right)-F(\bar{x}, \bar{y})}{t_{n}} \geq 0
\end{aligned}
$$

Thus,

$$
F_{d}^{+}\left((\bar{x}, \bar{y}),\left(v_{1}, v_{2}\right)\right) \geq 0, \text { for all }\left(v_{1}, v_{2}\right) \in T(\Omega,(\bar{x}, \bar{y})) .
$$

- On the one hand, using the upper semiregularity of $\partial^{*} F(\bar{x}, \bar{y})$ at $(\bar{x}, \bar{y})$, we get

$$
\sup _{\eta \in \partial^{*} F(\bar{x}, \bar{y})}\left\langle\eta,\left(v_{1}, v_{2}\right)\right\rangle \geq 0, \text { for all }\left(v_{1}, v_{2}\right) \in T(\Omega,(\bar{x}, \bar{y})) .
$$

From this, we can conclude easily from the calculus of the support functions that

$$
0 \in \overline{\overline{c o}\left(\partial^{*} F(\bar{x}, \bar{y})\right)+[T(\Omega,(\bar{x}, \bar{y}))]^{-}} .
$$

- On the other hand, the nonsmooth generalized Guignard constraint qualification implies that

$(0,0) \in c l\left[+\operatorname{cl} \operatorname{cone}\left\{\bigcup_{i \in I(\bar{x}, \bar{y})} \operatorname{conv} \partial^{*} g_{i}(\bar{x}, \bar{y}) \cup \bigcup_{j \in J(\bar{x}, \bar{y})}^{\operatorname{clconv}\left(\partial^{*} F(\bar{x}, \bar{y})\right)} \operatorname{conv} \partial^{*} G_{j}(\bar{x}, \bar{y}) \cup(\partial f(\bar{x}, \bar{y})-\partial V(\bar{x}) \times\{0\})\right\}\right]$

which implies that

$$
(0,0) \in \operatorname{conv} \partial^{*} F(\bar{x}, \bar{y})+c l\left\{\sum_{i \in I(\bar{x}, \bar{y})} \operatorname{cone} \partial^{*} g_{i}(\bar{x}, \bar{y})+\sum_{j \in J(\bar{x}, \bar{y})} \operatorname{cone} \partial^{*} G_{j}(\bar{x}, \bar{y})+\operatorname{cone}(\partial f(\bar{x}, \bar{y})-\partial V(\bar{x}) \times\{0\})\right\}
$$

By Proposition 2.3.15 [2], one has :

$$
\partial f(x, y) \subset \partial_{x} f(x, y) \times \partial_{y} f(x, y) .
$$


- Applying Theorem 8 [15] (its inner semicompact counterpart), we get

$$
\left.\partial V(\bar{x}):=\left[\bigcup_{y \in \psi(\bar{x})}\left\{\bigcup_{\left(\lambda_{1}, \cdots, \lambda_{m_{1}}\right) \in \Lambda(\bar{x}, y)} \partial_{x} f(\bar{x}, y)+\sum_{i \in I} \lambda_{i} \partial_{x} g_{i}(\bar{x}, y)\right)\right\}\right]
$$

where

$$
\Lambda(\bar{x}, y)=\left\{\left(\lambda_{1}, \cdots, \lambda_{m_{1}}\right) \in \mathbb{R}^{m_{1}}: 0 \in \partial_{y} f(\bar{x}, y)+\sum_{i \in I} \lambda_{i} \partial_{y} g_{i}(\bar{x}, y), \lambda_{i} \geq 0, \lambda_{i} g_{i}(\bar{x}, y)=0, i \in I\right\} .
$$

Necessary optimality conditions (4), (5) and (6) follow from (7), (9) and (10).

Theorem 2. Let $\bar{u}=(\bar{x}, \bar{y}) \in C$ be a local weak efficient solution of $(P)$. Assume that $F$ admits a bounded (USRCF) $\partial^{*} F(\bar{u})$ at $\bar{u}$, that $G_{j}, j \in J, g_{i}, i \in I$, admit (UCFs) $\partial^{*} G_{j}(\bar{u}), \partial^{*} g_{i}(\bar{u})$, respectively at $\bar{u}$. Suppose that the nonsmooth generalized Guignard constraint qualification holds at $\bar{u}$, that the solution-set-mapping of $\psi$ is inner semicontinuous at $(\bar{x}, \bar{y})$ and that $(\bar{x}, \bar{y})$ is lower-level regular. Then, there exist $\pi \geq 0, \xi \geq 0, \mu \geq 0$ and $\left(\lambda_{1}^{*}, \cdots, \lambda_{q}^{*}\right) \in \mathbb{R}_{+}^{q}$ such that

$$
\begin{array}{r}
0 \in \operatorname{conv} \partial_{x}^{*} F(\bar{x}, \bar{y})+\sum_{j \in J(\bar{x}, \bar{y})} \pi_{j} \partial_{x}^{*} G_{j}(\bar{x}, \bar{y})+\sum_{i \in I(\bar{x}, \bar{y})} \xi_{i} \partial_{x}^{*} g_{i}(\bar{x}, \bar{y}) \\
+\mu\left(\partial_{x} f(\bar{x}, \bar{y})-\partial_{x} f(\bar{x}, \bar{y})-\sum_{i \in I} \lambda_{i}^{*} \partial_{x} g_{i}(\bar{x}, \bar{y})\right) \\
0 \in \operatorname{conv} \partial_{y}^{*} F(\bar{x}, \bar{y})+\sum_{j \in J(\bar{x}, \bar{y})} \pi_{j} \partial_{y}^{*} G_{j}(\bar{x}, \bar{y})+\sum_{i \in I(\bar{x}, \bar{y})} \xi_{i} \partial_{y}^{*} g_{i}(\bar{x}, \bar{y})+\mu \partial_{y} f(\bar{x}, \bar{y})
\end{array}
$$

and

$$
0 \in \partial_{y} f(\bar{x}, \bar{y})+\sum_{i \in I} \lambda_{i}^{*} \partial_{y} g_{i}(\bar{x}, \bar{y}) \quad, \quad \lambda_{i}^{*} g_{i}(\bar{x}, \bar{y})=0
$$

Proof. Under the inner semicontinuity assumption on $S$, instead of (9), one gets

$$
\partial V(\bar{x}):=\bigcup_{\left(\lambda_{1}, \cdots, \lambda_{m_{1}}\right) \in \Lambda(\bar{x}, y)}\left(\partial_{x} f(\bar{x}, \bar{y})+\sum_{i \in I} \lambda_{i} \partial_{x} g_{i}(\bar{x}, \bar{y})\right)
$$

where

$\Lambda(\bar{x}, \bar{y})=\left\{\left(\lambda_{1}, \cdots, \lambda_{m_{1}}\right) \in \mathbb{R}^{m_{1}}: 0 \in \partial_{y} f(\bar{x}, \bar{y})+\sum_{i \in I} \lambda_{i} \partial_{y} g_{i}(\bar{x}, \bar{y}), \lambda_{i} \geq 0, \lambda_{i} g_{i}(\bar{x}, \bar{y})=0, i \in I\right\}$.

Using the same argument as in Theorem (1), one gets (11), (12) and (13).

Remark 6. The necessary optimality conditions we found are in fact generalized corrections of those given in [11]. Since Lemma 5.2 in [11] is wrong (setting $A=R+$ and $B=\{-1\}$ yields a simple counterexample) and since it is an integral part of the proof of Theorem 5.1, the necessary optimality conditions obtained by Kohli [11], as well as their proofs, are false.

\section{Conclusion}

In this note, we investigate a bilevel optimization problem $(P)$. Our approach consists of reformulating the problem using the optimal value function of the lower level problem and after investigating necessary optimality conditions of $(P)$. Using a nonsmooth generalized Guignard constraint qualification, one gives necessary optimality conditions in terms of convexificators and Karush-Kuhn-Tucker multipliers. 
Optimality conditions for a bilevel optimization problem in terms of KKT multipliers and convexificators 335

\section{Acknowledgement}

This work has been supported by the Alexander-von-Humboldt foundation.

\section{References}

[1] Bard, J. F. (1998). Practical Bilevel Optimization-Algorithms and Applications. Nonconvex Optimization and Its Applications, 30, 232-268. Springer: Boston. doi: 10.1007/978-1-4757-2836-1_7

[2] Clarke, F. H. (1990). Optimization and Nonsmooth Analysis. Classics in Applied Mathematics. Monograph published by SIAM. doi: 10.1137/1.9781611971309

[3] Dempe, S. (2002). Foundations of Bilevel Programming. Nonconvex Optimization and Its Applications, 61. Springer: Boston. doi: 10.1007/b101970

[4] Dempe, S. (2003). Annotated Bibliography on Bilevel Programming and Mathematical Programs with Equilibrium Constraints. Optimization, 52(3), 333-359. doi: 10.1080/0233193031000149894

[5] Dempe, S., Dutta, J. and Mordukhovich, B. S. (2007). New necessary optimality conditions in optimistic bilevel programming. Optimization, 56(5-6), 577-604. doi: 10.1080/02331930701617551

[6] Dempe, S. and Gadhi, N. (2007). Necessary optimality conditions for bilevel set optimization problems. Journal of Global Optimization, 39(4), 529-542. doi: 10.1007/s10898-007-9154-0

[7] Demyanov, V. F. and Jeyakumar, V. (1997). Hunting for a Smaller Convex Subdifferential. Journal of Global Optimization, 10(3), 305-326. doi: 10.1023/A:1008246130864

[8] Dutta, J. and Chandra, S. (2002). Convexifactors, Generalized Convexity, and Optimality Conditions. Journal of Optimization Theory and Applications, 113(1), 41-64. doi: 10.1023/a:1014853129484

[9] Göpfert, A., Riahi, H., Tammer, C. and Zàlinescu, C. (2003). Variational Methods in Partially Ordered Spaces. Springer-Verlag: Canadian Mathematical Society. doi: /10.1007/b97568

[10] Jeyakumar, V. and Luc, D. T. (1999). Nonsmooth Calculus, Minimality, and Monotonicity of Convexifactors. Journal of Optimization Theory and Applications, 101(3), 599-621. doi: 10.1023/a:1021790120780

[11] Kohli, B. (2012). Optimality Conditions for Optimistic Bilevel Programming Problem Using Convexifactors. Journal of Optimization Theory and Applications. 152(3), 632-651. doi: 10.1007/s10957-011-9941-0

[12] Luderer, B. (1983). Über der Äquivalenz nichtlinearer Optimierungsaufgaben. Technical Report, Technische Universität Karl-Marx-Stadt.

[13] Michel, P., Penot, J. P. and Brezis, H. (1992). A generalized derivative for calm and stable functions. Differential and Integral Equations, 5(2), 433-454. https://projecteuclid.org/euclid. die/1371043981

[14] Mordukhovich, B. S. and Nam, N. M. (2005). Variational Stability and Marginal Functions via Generalized Differentiation. Mathematics of Operations Research, 30(4), 800-816. doi: 10.1287/moor.1050.0147

[15] Mordukhovich, B. S., Nam, N. M., and Yen, N. D. (2009). Subgradients of marginal functions in parametric mathematical programming. Mathematical Programming, 116(1-2), 369-396. doi: 10.1007/s10107-007-0120-x

[16] Mordukhovich, B. S. and Shao, Y. (1995). On Nonconvex Subdifferential Calculus in Banach Spaces. Journal of Convex Analysis, 2(1-2), 211-227. http://www.heldermann.de/JCA/jca02.htm

[17] Ren, A. (2018). Solving the General Fuzzy Random Bilevel Programming Problem Through $M e$ Measure-Based Approach. IEEE Access, 6, 25610-25620. doi: 10.1109/access.2018.2828706

[18] Singh, V. P. and Chakraborty, D. (2017). Solving bi-level programming problem with fuzzy random variable coefficients. Journal of Intelligent and Fuzzy Systems, 32(1), 521-528. doi: 10.3233/jifs152354

[19] Ye, J. J. and Zhu, D. L. (1995). Optimality conditions for bilevel programming problems. Optimization, 33(1), 9-27. doi: 10.1080/02331939508844060

[20] Zhang, R. (1994). Problems of Hierarchical Optimization in Finite Dimensions. SIAM Journal on Optimization, 4(3), 521-536. doi: 10.1137/0804029 